\title{
BMJ Open Consensus on the exercise and dosage variables of an exercise training programme for chronic non-specific neck pain: protocol for an international e-Delphi study
}

\author{
Jonathan Price (D) , ${ }^{1}$ Alison Rushton (D) , ${ }^{2}$ Vasileios Tyros, ${ }^{3}$ Nicola R Heneghan (D) ${ }^{2}$
}

To cite: Price J, Rushton A, Tyros V, et al. Consensus on the exercise and dosage variables of an exercise training programme for chronic non-specific neck pain: protocol for an international e-Delphi study. BMJ Open 2020;10:e037656. doi:10.1136/ bmjopen-2020-037656

- Prepublication history and additional material for this paper are available online. To view these files, please visit the journal online (http://dx.doi. org/10.1136/bmjopen-2020037656).

Received 17 February 2020 Revised 15 April 2020 Accepted 23 April 2020

\section{Check for updates}

(c) Author(s) (or their employer(s)) 2020. Re-use permitted under CC BY-NC. No commercial re-use. See rights and permissions. Published by BMJ.

${ }^{1}$ Musculoskeletal Physiotherapy Services, Birmingham Community Healthcare NHS Foundation Trust, Birmingham, UK

${ }^{2}$ Centre of Precision

Rehabilitation for Spinal Pain (CPR Spine), School of Sport, Exercise and Rehabilitation Sciences, University of Birmingham, Birmingham, UK ${ }^{3}$ Edgbaston Physiotherapy Clinic, Birmingham, UK

Correspondence to

Dr Nicola R Heneghan;

n.heneghan@bham.ac.uk

\section{ABSTRACT}

Introduction Clinical guidelines and systematic reviews recommend exercise in the management of chronic nonspecific neck pain. Although exercise training programmes that consist of both motor control exercise and exercises for the superficial cervical muscles (segmental exercises) are effective, the exercise variables including dosage vary considerably across trials or are poorly reported. This study aims to gain expert consensus on these exercise variables so that they can be described clearly using intervention reporting checklists to inform clinical practice and future clinical trials.

Methods and analysis This protocol for an international Delphi study is informed by the Guidance on Conducting and REporting DElphi Studies recommendations and published to ensure quality, rigour and transparency. The study will consist of three rounds using anonymous online questionnaires. Expert exercise professionals (physiotherapists, strength and conditioning coaches and so on) and academics in neck pain management will be identified through literature searches, peer referral and social media calls for expression of interest. In round 1, participants will answer open-ended questions informed by intervention and exercise reporting checklists. Responses will be analysed thematically by two independent reviewers. In round 2, participants will rate their level of agreement with statements generated from round 1 and previous clinical trials using a 5-point Likert scale where $1=$ strongly disagree and $5=$ strongly agree. In round 3, participants will re-rate their agreement with statements that achieved consensus in round 2 . Statements reaching consensus among participants must meet progressively increased a priori criteria at rounds 2 and 3 , measured using descriptive statistics: median, IQR and percentage agreement. Inferential statistics will be used to evaluate measures of agreement between participants (Kendall's coefficient of concordance) and stability between rounds (Wilcoxon rank-sum test). Statements achieving consensus in round 3 will provide expert recommendations of the key exercise and dosage variables in the management of chronic non-specific neck pain.

Ethics and dissemination Ethical approval was provided by the University of Birmingham Ethics Committee (Ref:ERN_19-1857). Results will be
Strengths and limitations of this study

- This Delphi protocol is informed by the Guidance on Conducting and REporting DElphi Studies recommendations.

- Expert eligibility is predefined and includes international and multiprofessional representation.

- This protocol states definitions and a priori criteria for consensus, agreement and stability.

- The study will use a systematic consensus process to provide expert recommendations on the exercise and dosage variables of an exercise training programme for chronic non-specific neck pain that can be used in clinical practice and future clinical trials.

- The study results will be specific to chronic nonspecific neck pain rehabilitation, limiting the external validity to other musculoskeletal conditions.

disseminated through peer-reviewed publications and conference presentations.

\section{INTRODUCTION}

Chronic non-specific neck pain (CNSNP) affects 289 million people worldwide with increasing prevalence. ${ }^{12}$ The subsequent disability is significant resulting in CNSNP being considered one of the leading causes of years lived with disability. ${ }^{2}$ Despite multiple guidelines and systematic reviews informing clinical practice, ${ }^{3-9}$ patient outcomes are suboptimal, reflected by the increasing rank of CNSNP's cause for global disabilityadjusted life years. ${ }^{10-12}$

One explanation for poor patient outcomes is the vague recommendations of 'exercise' or 'strengthening exercise' from clinical guidelines and systematic reviews that inform clinical practice. Furthermore, exercise dosage recommendations (sets, repetitions, load, frequency and so on) are lacking and considered a research priority. ${ }^{13}$ Several 
trials have demonstrated small to very large short-term effects on pain and disability when using exercise training programmes that combine submaximal effort exercises for the deep cervical muscles to improve co-ordination and sequential spinal control (motor control exercises) and exercises for the superficial cervical muscles to improve the ability of the neck to produce, transfer and absorb force (segmental exercises) ${ }^{14-20}$ Although packages using a combination of motor control and segmental exercise appear promising, the optimal dosage and longterm effectiveness of this exercise training programme are unknown and require evaluation through an adequately powered low risk of bias clinical trials.

The Medical Research Council recommends complex interventions such as exercise training programmes to be defined prior to clinical trials. ${ }^{21}$ The Consensus on Exercise Reporting Template (CERT) ${ }^{22}$ and the Template for Intervention Description and Replication (TIDieR) ${ }^{23}$ are reporting checklists that aid in defining and reporting the design of exercise interventions. A key component of each reporting checklist is exercise training variables such as progressive overload, specificity, exercise selection order and dosage, as manipulation of these variables results in different performance and physical outcomes. ${ }^{24}{ }^{25}$ To date, the exercise and dosage variables of an exercise training programme of motor control and segmental exercise for CNSNP cannot be reported in accordance with CERT or TIDieR due to significant heterogeneity or poor reporting of previous trials. ${ }^{14-20}$ Consensus on these variables will enable a clearly defined exercise intervention for use in clinical trials and clinical practice.

\section{Aims}

The aim of this e-Delphi study is twofold. Initially, we will obtain exercise and academic professional expert opinion on the exercise and dosage variables of an exercise training programme consisting of motor control and segmental exercise for the management of CNSNP. We will then conduct a systematic process to gain consensus on the exercise and dosage variables reported to inform future research and clinical practice.

\section{METHODOLOGY}

\section{Justification of Delphi methodology}

Exploring exercise variables through qualitative research methodologies such as interviews and focus groups would embrace the diversity of opinion between experts providing rich detail and a deeper understanding, but this diversity maybe problematic when defining an intervention. ${ }^{26}$ As heterogeneity of motor control and segmental exercise variables already exists across trials, a convergence of information is required. Research methodologies such as Delphi techniques, consensus development conferences and nominal group techniques can all achieve consensus. ${ }^{27} 28$ The Delphi method is a systematic approach to achieving consensus among experts through the independent completion of sequential questionnaires

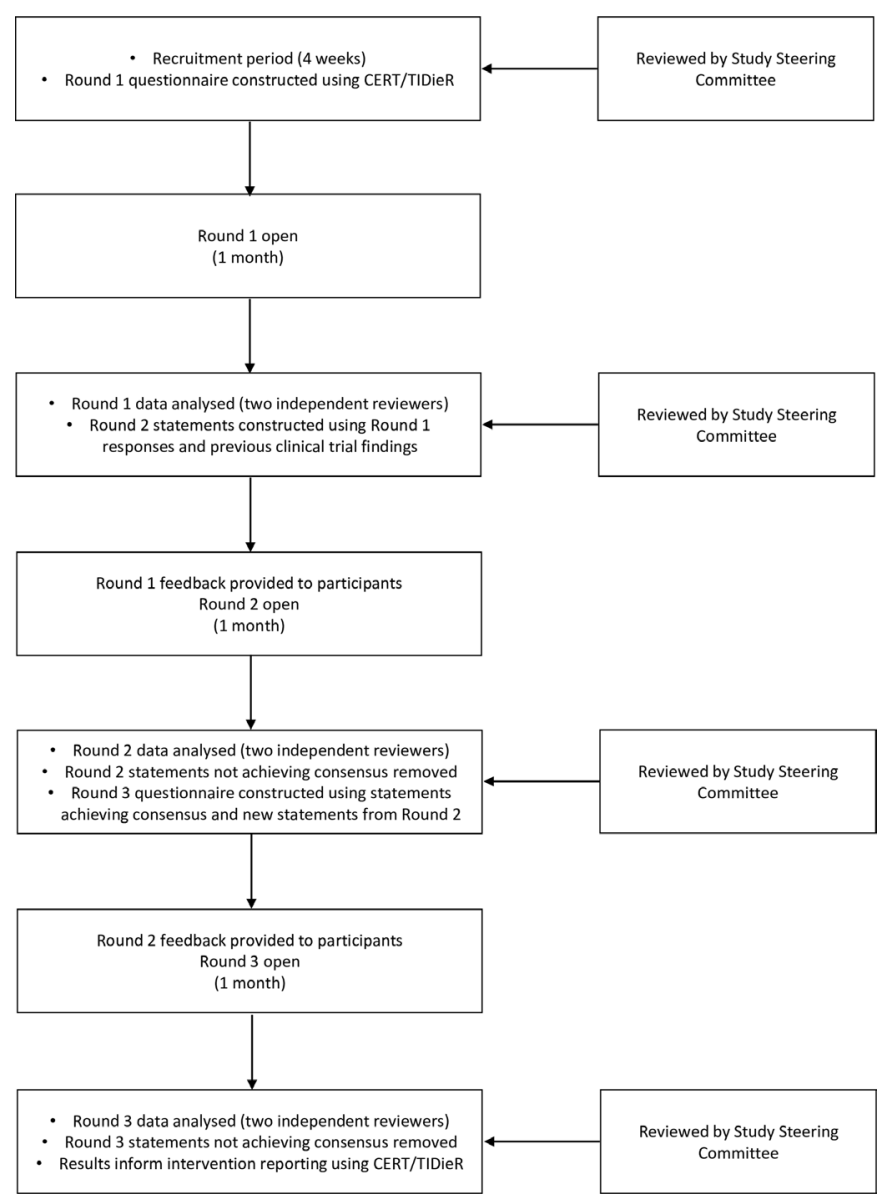

Figure 1 Delphi study procedures. CERT, Consensus on Exercise Reporting Template; TIDieR, Template for Intervention Description and Replication.

that are refined on feedback resulting in a convergence of opinion and eventual consensus. ${ }^{29}$ A Delphi method is advantageous over other forms of consensus techniques owing to (1) independent and anonymous participation reducing peer pressure and other extrinsic factors present in group techniques that cause subject bias ${ }^{30}{ }^{31}$; (2) controlled feedback between rounds encouraging consensus by providing participants with the opportunity to refine their opinions ${ }^{29}$; (3) using experts with a range of knowledge and experience to improve content validity and response rates ${ }^{32-34}$; and (4) electronic questionnaires removing geographical limitations. ${ }^{29}$

\section{Design}

This protocol is informed by Guidance on Conducting and REporting DElphi Studies (CREDES) ${ }^{35}$ (online supplementary file 1) and other recommended criteria. ${ }^{36}$ As no register exists for Delphi research, the protocol has been published to ensure quality, rigour and transparency. The three-round e-Delphi is summarised in figure $1 .{ }^{27}$ Data collection is planned between March and August 2020. All rounds will be completed electronically and anonymously using REDCap, a secure web application for building and managing online surveys. ${ }^{37} 38$ Round 1 will be used to generate statements on exercise and dosage variables for 
both motor control and segmental exercises. Experts will rate their agreement with statements in rounds 2 and 3 using a 5-point Likert scale. Three rounds are commonly cited to be sufficient to achieve consensus. ${ }^{27}$ Statements that achieve consensus in round 3 will be used to describe the key exercise and dosage variables for a CNSNP exercise training programme which includes motor control and segmental exercises.

\section{Expert eligibility and sample}

A purposive sampling method will be used to recruit a range of experts in CNSNP exercise prescription. It is recommended that a heterogeneous sample is used representing a spectrum of opinions ${ }^{29}$ and therefore experts will be recruited from two distinct groups:

- Exercise professionals: any professional who uses exercise to manage neck pain will be considered (eg, physiotherapists, strength and conditioning coaches, osteopaths and chiropractors). Eligible participants will have a relevant postgraduate qualification or $>5$ years of sports or musculoskeletal experience. Experts will treat $\geq 5$ patients with CNSNP per month using exercise. Experts will be identified through existing professional networks and social media/internetbased searching.

- Academics: eligible academics will have $\geq 2$ peerreviewed publications focused on the use of exercise in the management of CNSNP in the past 10 years. ${ }^{39}$ Academics will be identified through CNSNP systematic reviews/randomised clinical trials published indexed in PubMed and Expertscape searches. ${ }^{13}$

Experts will be recruited worldwide, aged 18 or above, able to read and write English and willing to participate. They will be invited to participate by the lead author (JP) through email. Recruitment will be maximised by encouraging identified experts to snowball the invitation with other suitable participants and calls for expressions of interest on social media. ${ }^{41}$ Upon experts confirming their interest and eligibility, they will be provided with a link to the Research Electronic Data Capture (REDCap) system at the University of Birmingham, where a Participant Information Sheet (online supplementary file 2) and Consent Form will be hosted. ${ }^{37} 38$ The Participant Information Sheet will clarify study procedures, eligibility criteria, assure anonymity and explain the withdrawal process. Participants may withdraw at any time up until the data analysis of the Round 3 Questionnaire. Due to the nature of the Delphi process, responses will be used up to the point of withdrawal. Participants will be able to withdraw from the study by contacting the Principal Investigator or Primary Academic Supervisor. Consent will be obtained electronically through REDCap. ${ }^{37} 38$ Recruitment will continue for 4 weeks with a reminder sent at week 2. Should there be no contact within the 4 weeks then no further communication will be sent. ${ }^{42}$

There is no universal guide to sample size in Delphi studies, and expert panels have ranged from 4 to 3000 participants. ${ }^{43}$ Previous Delphi studies with an aim of intervention development typically achieved consensus with responses from 10 to 27 experts in the final round ${ }^{39} 44-47$ and therefore, a conservative estimate of 27 final responses is required. Assuming a response rate of $70 \%$, a minimum of 40 experts are required to complete the consent form to ensure at least 27 responses in round $3{ }^{29}$ To prevent over-representation from one expert group, recruitment will be monitored to achieve an approximate 50/50 split between exercise and academic professionals.

\section{Procedure}

Round 1

The objectives of round 1 will be to obtain participant demographic data and generate statements on exercise and dosage variables based on expert opinion. Participants will complete the Participant Details Form collecting information on professional background, highest qualification, primary country of work, work setting, H-index, publication count, years qualified and grade of clinical work. The Round 1 Questionnaire (online supplementary file 3) will consist of open-ended questions informed by CERT and TIDieR. ${ }^{22}{ }^{23}$ Open-ended questions improve the content validity as statements are generated by expert opinion. ${ }^{29}{ }^{31}$ Statements generated from previous clinical trials ${ }^{14-20}$ will be included in round 2 , rather than round 1 to allow participants to provide their expert opinion without bias from the literature, thereby reducing experimenter bias. ${ }^{34}$ The questions will ask participants to identify the exercise and dosage variables they consider important when prescribing exercise for CNSNP. They will then be asked to list and explain what patients or other factors may affect or inform their reasoning when prescribing the exercise and dosage variables that they identified. Participants will be asked to answer open-ended questions for both motor control and segmental exercises independently with definitions of both subgroups of exercise provided for clarity (online supplementary file 3). ${ }^{48}$ Participants will have the opportunity to provide general comments at the end. The Round 1 Questionnaire (online supplementary file 3) was piloted for feedback on readability, relevance and appropriateness through the Study Steering Group and edited accordingly. Round 1 will be open for 1 month with email reminders, including the withdrawal process, being provided at weeks 1 and $3{ }^{49}$

\section{Round 2}

The objectives of round 2 are to evaluate consensus on statements regarding exercise and dosage variables and to identify any further statements. Participants will be provided with feedback explaining how statements were generated from round 1 and then asked to rate their agreement with the statements using a 5-point Likert scale where $1=$ strongly disagree and $5=$ strongly agree ${ }^{49}$ A 5-point scale is preferred as it possesses acceptability psychometric properties while being quick and easy for participants to reduce frustration and demotivation. ${ }^{50}$ An open text box will be included for each statement for 
any additional comments or further statement generation. All comments will be analysed by the study team and reviewed by the Study Steering Group. All participants will be invited to round 2, including those who did not complete round 1, provided they have not withdrawn from the study. This provides the opportunity for participants to continue their involvement who were unable to complete previous rounds due to time or other commitments. ${ }^{29}$ As per round 1, the Round 2 Questionnaire will remain active for 4 weeks with email reminders sent at weeks 1 and 3 .

\section{Round 3}

The objective of round 3 is to strengthen consensus on statements regarding exercise and dosage variables. The Round 3 Questionnaire will include feedback from round 2 using descriptive statistics, promoting reflection before completing the final questionnaire. In round 3, participants will be asked to rate their agreement with the statements achieving consensus from round 2 using the same 5-point Likert scale. ${ }^{39}$ Statements that do not achieve consensus in round 2 will be discarded. A free-text box will be provided for participants to clarify responses but the generation of new statements will not be encouraged. All participants will be invited to participate in round 3 , which will again remain active for 4 weeks with email reminders sent at weeks 1 and 3 .

\section{Data analysis}

Quantitative and qualitative data will be inputted into IBM SPSS Statistics V.25 and QSR International's NVivo V.12 Plus software, respectively, for analyses. ${ }^{5152}$ Data will be analysed independently by two researchers (JP and VT) at each round. The complete agreement between researchers is required for statements to be included, with disagreements resolved by discussion. ${ }^{53}$ The Study Steering Group will review the data at each stage for feedback and editing before dissemination.

\section{Round 1}

Qualitative data will be examined using a theoretical thematic analysis to generate statements under themes preidentified from CERT/TIDieR and then examined inductively for any new themes. ${ }^{26}{ }^{54}$ Original wording from one expert that best represents the wording across participants with similar statements will be used where possible and all other statements will be discarded. ${ }^{34}$ Statements generated from previous clinical trial findings not identified from participant responses will also be included ${ }^{14-20}$ For any statement to be included, it must be described at least once by any participant or via previous clinical trials; therefore, any stand-alone statements will be kept and included. The Round 2 Questionnaire will be constructed using the statements generated.

\section{Round 2}

Qualitative data will be analysed using thematic analysis for the emergence of any new statements. Descriptive and inferential statistics will be used to evaluate agreement and consensus (table 1). Any statements not achieving the a priori criteria for consensus will be discarded (median $\geq 3$; IQR $\leq 1.5$; percentage agreement $\geq 60 \%$ ).

\section{Round 3}

Descriptive and inferential statistics will evaluate consensus against a priori criteria (median $\geq 3.5$; IQR $\leq 1$; percentage agreement $\geq 70 \%$ ) (table 1 ). Statements achieving consensus after round 3 will be used to describe the key exercise and dosage variables of motor control and segmental exercise training programme. Statements that fail to achieve consensus in round 3 will be discarded.

\section{Consensus, agreement and stability}

A discrepancy exists as to the definitions and statistical measures of consensus and agreement within the literature. ${ }^{36535556}$ Some argue that consensus and agreement are interchangeable, ${ }^{55}$ whereas others recommend separate definitions. ${ }^{57} 58$ To ensure clarity, the following definitions will be used in this study:

- Consensus: the extent to which the group of experts share the same opinion..$^{55}$

- Agreement: a measure of inter-rater agreement where the rating of one expert can be predicted by the rating of another. ${ }^{59}$

- Stability: the consistency of responses between successive rounds. ${ }^{55} 57$

Consensus, agreement and stability will be assessed in each round using a combination of descriptive and

Table 1 Definitions and statistical measures of consensus, agreement and stability

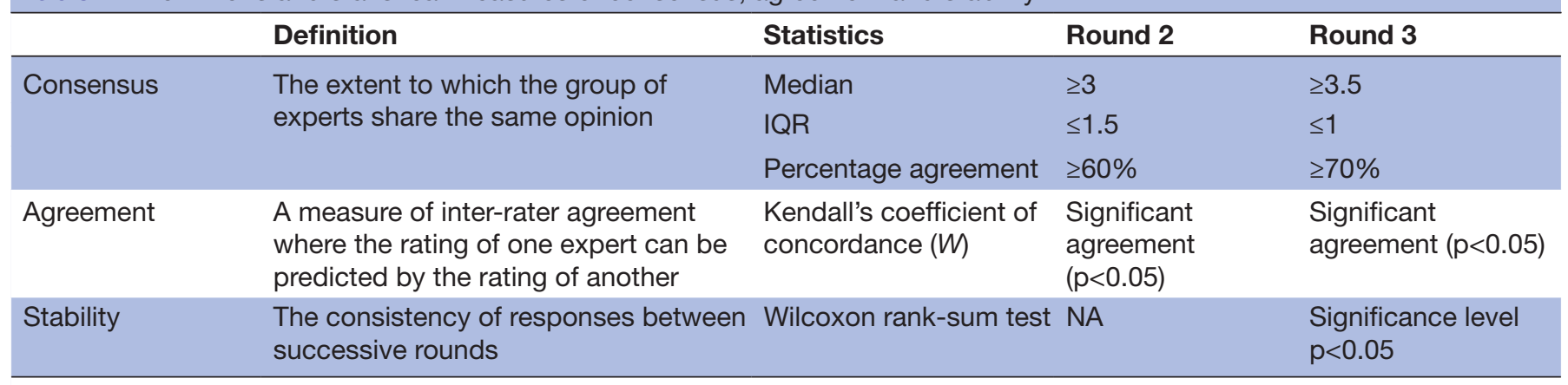


inferential statistics (table 1). ${ }^{365356}$ Consensus will be evaluated using descriptive statistics of central tendency and dispersion. As the Likert scale is considered an ordinal scale ${ }^{60}$ median and IQR will be used. ${ }^{5560}$ Percentage agreement, defined as the percentage of responses rated agree/strongly agree, will also be used to evaluate consensus among experts for each statement. ${ }^{39}{ }^{49}$ Progressively increased criteria will be used between rounds 2 and 3 to encourage convergence and strengthen overall consensus. ${ }^{39}{ }^{49}$ Agreement between experts across all items and within categories identified after round 1 will be evaluated using Kendall's coefficient of concordance $(W)$ where 0 is no agreement and 1 is perfect agreement. ${ }^{59}$ The stability of the responses between rounds 2 and 3 will be evaluated using the Wilcoxon rank-sum test. ${ }^{5561}$ Statistical significance will be set at $\mathrm{p}<0.05$.

\section{Data management}

All personal information and data will be kept secure from any third party using a password-protected computer during the study. Only members of the study team will have access to the study data. On completion of the study, the data will be kept securely for 10 years in the School of Sport, Exercise and Rehabilitation Sciences, University of Birmingham, UK before being securely destroyed in accordance with University guidelines.

\section{Study steering group}

The Study Steering Group will provide study oversight with members consisting of coauthors and patient/public, methodological and clinical expertise (table 2). The Study Steering Group will meet at key stages throughout the

Table 2 Study steering group members, backgrounds and roles

\begin{tabular}{|c|c|c|}
\hline Background & Professional title & Role \\
\hline Patient & NA & $\begin{array}{l}\text { Co-chair/patient } \\
\text { representative }\end{array}$ \\
\hline $\begin{array}{l}\text { Academic } \\
\text { nurse }\end{array}$ & Lecturer & $\begin{array}{l}\text { Co-chair/ } \\
\text { methodological } \\
\text { representative }\end{array}$ \\
\hline $\begin{array}{l}\text { Clinical } \\
\text { physiotherapist }\end{array}$ & $\begin{array}{l}\text { Consultant } \\
\text { physiotherapist }\end{array}$ & Clinical representative \\
\hline Patient & NA & Patient representative \\
\hline $\begin{array}{l}\text { Academic } \\
\text { physiotherapist }\end{array}$ & Senior lecturer & Primary supervisor \\
\hline $\begin{array}{l}\text { Academic } \\
\text { physiotherapist }\end{array}$ & $\begin{array}{l}\text { Reader in } \\
\text { musculoskeletal } \\
\text { sciences }\end{array}$ & Secondary supervisor \\
\hline $\begin{array}{l}\text { Clinical } \\
\text { physiotherapist }\end{array}$ & Physiotherapist & Co-investigator \\
\hline $\begin{array}{l}\text { Clinical } \\
\text { academic } \\
\text { trainee } \\
\text { physiotherapist }\end{array}$ & $\begin{array}{l}\text { Pre-doctoral } \\
\text { clinical academic } \\
\text { fellow }\end{array}$ & Principle investigator \\
\hline
\end{tabular}

NA, not applicable. study to provide feedback on questionnaire development, structure and clarity; aid in expert identification; review study results at each round and agree statement inclusion; review study conduct and aid in the dissemination of findings. Members of the Study Steering Group who are not coauthors will not have access to raw data or be able to influence the study process. Feedback and changes suggested by the Study Steering Group must be agreed between the study coauthors before implementation.

\section{Ethics}

Appropriate ethical approval has been granted from the University of Birmingham Ethics Committee (Ref: ERN_19-1857). Informed consent will be received from all participants before completing any questionnaires. They will be informed of the withdrawal process and that any feedback will be anonymised for privacy.

\section{Patient and public involvement}

The study was conceived from our clinical working with patients with spinal complaints over many years and their views used to highlight the relevance of this research. Two patients were involved in reviewing the findings of the original systematic review that underpinned this study, suggesting alternatively terminology that better reflects patient views. Our Study Steering Group patient representatives helped refine the research aim of this study as well as contributing to the design of Participant Information Sheets, expression of interest emails/social media posts and developing the Round 1 Questionnaire. It is anticipated that our patient representatives will continue to co-chair the Study Steering Group, review study results at each round and study conduct. Our patient representatives will be instrumental in future dissemination of findings to patient cohorts, as well as informing future fellowship applications for the lead author (JP). Patient and public involvement in the full study will be reported using the Guidance for Reporting Involvement of Patients and the Public2-short form (GRIPP2-SF) when disseminating the study results. ${ }^{62}$

\section{DISCUSSION}

This Delphi study will provide expert consensus on the exercise and dosage variables of an exercise training programme consisting of motor control and segmental exercises for CNSNP that could not be determined from the current literature. Conducting an e-Delphi allows the development of expert informed recommendations from a range of worldwide experts who can participate anonymously, which should be considered a strength. The expert eligibility criteria could be considered a limitation of the study as it may exclude experts in exercise prescription who see a small volume of patients experiencing neck pain. However, there is currently no clear guidelines as to how best define an expert. ${ }^{273563}$ The strict eligibility criteria used are important to ensure that findings are appropriate to CNSNP as it is currently unknown whether 
approaches to exercise are transferable between different musculoskeletal conditions. Future research will require the acceptability and feasibility of exercise and dosage variables to be evaluated by patient and physiotherapists focus groups. Results will inform the development of an intervention that will be defined using CERT/TIDieR and evaluated in a low risk of bias, adequately powered clinical trial investigating long-term outcomes and optimal dosage.

Twitter Jonathan Price @PhysioPrice, Alison Rushton @abrushton and Nicola R Heneghan @HeneghanNicola

Acknowledgements This work was completed as part of an NIHR/HEE Predoctoral Clinical Academic Fellowship. Academic support provided from the University of Birmingham and the Centre of Precision Rehabilitation for Spinal Pain. The authors would like to thank the clinical, methodological and patient experts who have agreed to be members of the Study Steering Group.

Contributors JP: is the $\mathrm{Cl}$ leading the protocol development, analyses and dissemination. NRH and AR: supervisors for the study, ensuring rigour and quality. JP and VT: will independently complete data analysis. All authors have contributed to the design and development of the protocol and have contributed to manuscript draft; read, provided feedback and approved the final manuscript.

Funding This Delphi study did not receive any specific grant from any funding agency in the public, commercial or not-for-profit sectors. It will be conducted as part of JPs MRes research project through the University of Birmingham. The MRes is a component of JP's academic training funded through the Health Education England/National Institute of Health Research's Pre-doctoral Clinical Academic Fellowship.

Competing interests None declared.

Patient and public involvement Patients and/or the public were involved in the design, or conduct, or reporting, or dissemination plans of this research. Refer to the Methods section for further details.

Patient consent for publication Not required.

Provenance and peer review Not commissioned; externally peer reviewed.

Open access This is an open access article distributed in accordance with the Creative Commons Attribution Non Commercial (CC BY-NC 4.0) license, which permits others to distribute, remix, adapt, build upon this work non-commercially, and license their derivative works on different terms, provided the original work is properly cited, appropriate credit is given, any changes made indicated, and the use is non-commercial. See: http://creativecommons.org/licenses/by-nc/4.0/.

\section{ORCID iDs}

Jonathan Price http://orcid.org/0000-0002-5505-1564

Alison Rushton http://orcid.org/0000-0001-8114-7669

Nicola R Heneghan http://orcid.org/0000-0001-7599-3674

\section{REFERENCES}

1 Andersen LL, Mortensen OS, Hansen JV, et al. A prospective cohort study on severe pain as a risk factor for long-term sickness absence in blue- and white-collar workers. Occup Environ Med 2011;68:590-2.

2 James SL, Abate D, Abate KH, et al. Global, regional, and national incidence, prevalence, and years lived with disability for 354 diseases and injuries for 195 countries and territories, 1990-2017: a systematic analysis for the global burden of disease study 2017 . Lancet 2018;392:1789-858.

3 Bier JD, Scholten-Peeters WGM, Staal JB, et al. Clinical practice guideline for physical therapy assessment and treatment in patients with nonspecific neck pain. Phys Ther 2018;98:162-71.

4 Blanpied PR, Gross AR, Elliott JM, et al. Neck pain: revision 2017. J Orthop Sports Phys Ther 2017;47:A1-83.

5 Bertozzi L, Gardenghi I, Turoni F, et al. Effect of therapeutic exercise on pain and disability in the management of chronic nonspecific neck pain: systematic review and meta-analysis of randomized trials. Phys Ther 2013;93:1026-36.

6 Chen X, Coombes BK, Sjøgaard G, et al. Workplace-Based interventions for neck pain in office workers: systematic review and meta-analysis. Phys Ther 2018;98:40-62.
7 Gross A, Kay TM, Paquin J-P, et al. Exercises for mechanical neck disorders. Cochrane Database Syst Rev 2015;1:CD004250.

8 Louw S, Makwela S, Manas L, et al. Effectiveness of exercise in office workers with neck pain: a systematic review and metaanalysis. S Afr J Physiother 2017;73:392.

9 Martin-Gomez C, Sestelo-Diaz R, Carrillo-Sanjuan V, et al. Motor control using cranio-cervical flexion exercises versus other treatments for non-specific chronic neck pain: a systematic review and meta-analysis. Musculoskelet Sci Pract 2019;42:52-9.

10 Murray CJL, Vos T, Lozano R, et al. Disability-adjusted life years (DALYs) for 291 diseases and injuries in 21 regions, 1990-2010: a systematic analysis for the global burden of disease study 2010. Lancet 2012;380:2197-223.

11 , Murray CJL, Barber RM, et al, GBD 2013 DALYs and HALE Collaborators. Global, regional, and national disability-adjusted life years (DALYs) for 306 diseases and injuries and healthy life expectancy (HALE) for 188 countries, 1990-2013: quantifying the epidemiological transition. Lancet 2015;386:2145-91.

$12 \mathrm{Kyu} \mathrm{HH}$, Abate D, Abate KH, et al. Global, regional, and national disability-adjusted life-years (DALYs) for 359 diseases and injuries and healthy life expectancy (HALE) for 195 countries and territories, 1990-2017: a systematic analysis for the global burden of disease study 2017. Lancet 2018;392:1859-922.

13 Silva PV, Costa LOP, Maher CG, et al. The new agenda for neck pain research: a modified Delphi study. J Orthop Sports Phys Ther 2019;49:666-74.

14 Bobos P, Billis E, Papanikolaou D-T, et al. Does deep cervical flexor muscle training affect pain pressure thresholds of myofascial trigger points in patients with chronic neck pain? A prospective randomized controlled trial. Rehabil Res Pract 2016;2016:6480826:1-8.

15 Bobos P, Papanikolaou T, Koutsojannis C, et al. Is deep cervical neck flexors' training more effective than General neck exercises or advice in patients with chronic neck pain? A prospective randomized controlled trial. Man Ther 2016;25:e145-6.

16 Chiu TTW, Hui-Chan CWY, Chein G. A randomized clinical trial of TENS and exercise for patients with chronic neck pain. Clin Rehabil 2005;19:850-60.

17 Chiu TTW, Lam T-H, Hedley AJ. A randomized controlled trial on the efficacy of exercise for patients with chronic neck pain. Spine 2005;30:E1-7.

18 Falla D, Lindstrøm R, Rechter L, et al. Effectiveness of an 8-week exercise programme on pain and specificity of neck muscle activity in patients with chronic neck pain: a randomized controlled study. Eur J Pain 2013;17:1517-1528.

19 Gupta S, Sharma S, Kataria C. Craniocervical flexors endurance training: treatment approach for cervical spondylosis. J Physiother Occup Ther2010;4:76-81.

20 Borisut S, Vongsirinavarat M, Vachalathiti R, et al. Effects of strength and endurance training of superficial and deep neck muscles on muscle activities and pain levels of females with chronic neck pain. $J$ Phys Ther Sci 2013;25:1157-62.

21 Craig P, Dieppe P, Macintyre S, et al. Developing and evaluating complex interventions: new guidance. London: Medical Research Council, 2019.

22 Slade SC, Dionne CE, Underwood M, et al. Consensus on exercise reporting template (CERT): explanation and elaboration statement. $\mathrm{Br}$ J Sports Med 2016;50:1428-37.

23 Hoffmann TC, Glasziou PP, Boutron I, et al. Better reporting of interventions: template for intervention description and replication (TIDieR) checklist and guide. BMJ 2014;348:g1687.

24 American College of Sports Medicine. American College of sports medicine position stand. progression models in resistance training for healthy adults. Med Sci Sports Exerc 2009;41:687-708.

25 Bird SP, Tarpenning KM, Marino FE. Designing resistance training programmes to enhance muscular fitness: a review of the acute programme variables. Sports Med 2005;35:841-51.

26 Braun V, Clarke V. Successful qualitative research: a practical guide for beginners. SAGE Publications, 2013.

27 Foth T, Efstathiou N, Vanderspank-Wright B, et al. The use of Delphi and nominal group technique in nursing education: a review. Int $J$ Nurs Stud 2016;60:112-20.

28 Black N, Murphy M, Lamping D, et al. Consensus development methods: a review of best practice in creating clinical guidelines. $J$ Health Serv Res Policy 1999;4:236-48.

29 Keeney S, McKenna H, Hasson F. The Delphi technique in nursing and health research. Wiley, 2010.

30 Dalkey $\mathrm{N}$, Helmer O. An experimental application of the Delphi method to the use of experts. Manage Sci 1963;9:458-67.

31 Goodman CM. The Delphi technique: a critique. J Adv Nurs $1987 ; 12: 729-34$. 
32 Murphy MK, Black NA, Lamping DL, et al. Consensus development methods, and their use in clinical Guideline development. Health Technol Assess 1998;2:1-88.

33 Keeney S, Hasson F, McKenna HP. A critical review of the Delphi technique as a research methodology for nursing. Int J Nurs Stud 2001;38:195-200.

34 Hasson F, Keeney S, McKenna H. Research guidelines for the Delph survey technique. J Adv Nurs 2000;32:1008-15.

35 Jünger S, Payne SA, Brine J, et al. Guidance on conducting and reporting Delphi studies (CREDES) in palliative care: recommendations based on a methodological systematic review. Palliat Med 2017;31:684-706.

36 Diamond IR, Grant RC, Feldman BM, et al. Defining consensus: a systematic review recommends methodologic criteria for reporting of Delphi studies. J Clin Epidemiol 2014;67:401-9.

37 Harris PA, Taylor R, Minor BL, et al. The REDCap Consortium: building an international community of software platform partners. $J$ Biomed Inform 2019;95:103208.

38 Harris PA, Taylor R, Thielke R, et al. Research electronic data capture (REDCap)--a metadata-driven methodology and workflow process for providing translational research informatics support. J Biomed Inform 2009;42:377-81.

39 Wiangkham T, Duda J, Haque MS, et al. Development of an active behavioural physiotherapy intervention (ABPI) for acute whiplashassociated disorder (WAD) II management: a modified Delphi study. BMJ Open 2016;6:e011764.

40 Alreni ASE, McLean SM, Demack S, Orthopaedic Proceedings. Managing non-specific neck pain: a national survey of current UK physiotherapy practice. 100-B, 2018.

41 Stephens G, O'Neill S, French HP, et al. A survey of physiotherapy practice (2018) in the United Kingdom for patients with greater trochanteric pain syndrome. Musculoskelet Sci Pract 2019;40:10-20.

42 Delbecq AL, Van de Ven AH, Gustafson DH. Group techniques for program planning: a guide to nominal group and Delphi processes. Scott, Foresman, 1975.

43 Cantrill JA, Sibbald B, Buetow S. The Delphi and nominal group techniques in health services research. International Journal of Pharmacy Practice 1996;4:67-74.

44 Conchin S, Carey S. The expert's guide to mealtime interventions - A Delphi method survey. Clin Nutr 2018;37:1992-2000.

$45 \mathrm{Kim}$ MJ, Sung E, Choi EY, et al. Delphi survey for designing a intervention research study on childhood obesity prevention. Korean J Fam Med 2017;38:284-90.

46 Wheatley A, Bamford C, Shaw C, et al. Developing an intervention for fall-related injuries in dementia (DIFRID): an integrated, mixedmethods approach. BMC Geriatr 2019;19:57.
47 Robinson KR, Leighton P, Logan P, et al. Developing the principles of chair based exercise for older people: a modified Delphi study. BMC Geriatr 2014;14:65.

48 Spencer S, Wolf A, Rushton A. Spinal-Exercise prescription in sport: classifying physical training and rehabilitation by intention and outcome. J Ath/ Train 2016;51:613-28.

49 Zambaldi M, Beasley I, Rushton A. Return to play criteria after hamstring muscle injury in professional football: a Delphi consensus study. Br J Sports Med 2017;51:1221-6.

50 Preston CC, Colman AM. Optimal number of response categories in rating scales: reliability, validity, discriminating power, and respondent preferences. Acta Psychol 2000;104:1-15.

51 IBM. SPSS Statistics [program. 25 version, 2017

52 NVIVO. NVivo program. 12 Plus version, 2018.

53 de Loë RC, Melnychuk N, Murray D, et al. Advancing the state of policy Delphi practice: a systematic review evaluating methodologica evolution, innovation, and opportunities. Technol Forecast Soc Change 2016;104:78-88.

54 Braun V, Clarke V. Using thematic analysis in psychology. Qual Res Psychol 2006;3:77-101.

55 von der Gracht HA. Consensus measurement in Delphi studies. Technol Forecast Soc Change 2012;79:1525-36.

56 Boulkedid R, Abdoul H, Loustau M, et al. Using and reporting the Delphi method for selecting healthcare quality indicators: a systematic review. PLoS One 2011;6:e20476.

57 Trevelyan EG, Robinson PN. Delphi methodology in health research: how to do it? Eur J Integr Med 2015;7:423-8.

58 Meijering JV, Kampen JK, Tobi H. Quantifying the development of agreement among experts in Delphi studies. Technol Forecast Soc Change 2013;80:1607-14.

59 Schmidt RC. Managing Delphi surveys using nonparametric statistical techniques. Decision Sciences 1997;28:763-74.

60 Hicks C. Chapter 4. The Nature of the Data. Research Methods for Clinical Therapists. In: Applied project design and analysis. Fourth edn. Churchill Livingstone, 2004.

61 Trevelyan EG, Turner WA, Robinson N. Developing an acupuncture protocol for treating phantom limb pain: a Delphi consensus study. Acupunct Med 2015;33:42-50.

62 Staniszewska S, Brett J, Simera I, et al. GRIPP2 reporting checklists: tools to improve reporting of patient and public involvement in research. BMJ 2017;358:j3453.

63 R Avella J. Delphi panels: research design, procedures, advantages, and challenges. International Journal of Doctoral Studies 2016;11:305-21. 